\title{
Short-term restoration of facial sensory loss by motor cortex stimulation in peripheral post-traumatic neuropathic pain
}

\author{
Denys Fontaine $\cdot$ Jean Louis Bruneto · \\ Hasna El Fakir · Philippe Paquis · \\ Michel Lanteri-Minet
}

Received: 26 December 2008/Accepted: 6 March 2009/Published online: 7 April 2009

(C) Springer-Verlag 2009

\begin{abstract}
We report a case in which motor cortex stimulation (MCS) improved neuropathic facial pain due to peripheral nerve injury and restored tactile and thermal sensory loss. A 66-year-old man developed intractable trigeminal neuropathic pain after trauma of the supraorbital branch of the Vth nerve, associated with tactile and thermal sensory loss in the painful area. MCS was performed using neuronavigation and transdural electric stimulation to localize the upper facial area on the motor cortex. One month after surgery, pain was decreased from 80/100 to 20/ 100 on visual analogic scale, and sensory discrimination improved in the painful area. Two months after surgery, quantitative sensory testing confirmed the normalization of thermal detection thresholds. This case showed that MCS could restore tactile and thermal sensory loss, resulting from peripheral nerve injury. Although the mechanisms leading to this effect remain unclear, this observation enhanced the hypothesis that MCS acts through modulation of the sensory processing.
\end{abstract}

Keywords Motor cortex stimulation .

Sensory thresholds · Quantitative sensory testing .

Neuropathic pain

D. Fontaine $(\bowtie) \cdot$ H. El Fakir · P. Paquis

Service de Neurochirurgie, Hospital Pasteur,

30 avenue de la voie romaine, 06000 Nice, France

e-mail: fontaine.d@chu-nice.fr

J. L. Bruneto - M. Lanteri-Minet

Département d'Evaluation et de Traitement de la Douleur,

CHU de Nice, UNSA, Nice, France

M. Lanteri-Minet

INSERM U929, Université Clermont-Ferrand 1,

Clermont-Ferrand, France

\section{Introduction}

Motor cortical stimulation (MCS) has been proposed to treat intractable chronic central and peripheral neuropathic pain $[1,2]$. The mechanisms underlying the pain control remain unclear. MCS can improve dysesthesias and allodynia in the painful area [3], but only few patients reported sensory changes after MCS [4] with lack of details concerning the delay of improvement in these patients. We report the case of a patient with post-traumatic peripheral neuropathic pain of the scalp in which MCS rapidly alleviated pain and sensory impairment, enhancing the hypothesis of modulation of the sensory processing.

\section{Case report}

A 66-year-old man had a cranial trauma in March 2004 with a wound of the right supra-orbital branch of the ophthalmic nerve (V1) inducing severe hypoesthesia of the anterior scalp. After 2 months, intense paroxysmal attacks of pain appeared in the area of sensory loss. The pain was precipitated from a trigger area located on right side of the bridge of the nose, not affected by the sensory loss. Progressively, intensity and frequency of painful paroxysms increased, and a lancinating constant pain developed. Initial treatment consisted in monotherapy with amitriptyline (rapidly stopped because of sedative effects) and various anti-epileptic drugs (gabapentin 2,400 $\mathrm{mg}$, carbamazepine $1,200 \mathrm{mg}$, oxcarbazepine $600 \mathrm{mg}$ ). Association of gabapentin and oxcarbazepine partially reduced the pain intensity. A first block of the supra-orbital branch of the ophthalmic nerve, using an association of local anesthetics and long-lasting steroids, partially alleviated the pain during 1 month. Two additional nerve blocks failed to confirm 
this initial improvement. Conventional trans-cutaneous electrical stimulation had no effect. As the pain became therapy-resistant, the patient was referred in August 2006 to discuss MCS.

Preoperative assessment showed a tactile and thermal sensory loss in the supra-orbital V1 territory (Fig. 1). The sensory thresholds for innocuous and noxious thermal stimulation were measured in the painful area by TSA 2001 apparatus (Medoc), using a Peltier device, according to previously described protocol [5], in comparison with the corresponding area on the uninjured side (Fig. 2). The tactile sensory loss was confirmed by the use of sensory testing monofilament (5.07/10 g filament). Pain was scored 80/100 on visual analogic scale (VAS). Patient assessment using HAD scale showed an emotional impact concerning anxious dimension but not depressive dimension (anxiety score $=10$, depression score $=4$ ).

Two quadripolar electrodes (Resume, Medtronic) were implanted epidurally over the left central sulcus over the area corresponding somatotopically to the right upper face, localized using MRI-based neuronavigation. After craniotomy, two parallel electrodes were placed just above the area where transdural electrical stimulation induced motor responses in the contralateral cheek. Electrodes were then connected to an internal generator (Synergy, Medtronic). The parameters used for chronic bipolar stimulation were: frequency $40 \mathrm{~Hz}$, amplitude $3 \mathrm{~V}$ and pulse width $210 \mathrm{~ms}$.

From the third post-operative day, the pain has sharply declined. One month after surgery, it was quoted 20/100 on the VAS. This pain relief concerned both paroxysmal and constant pain. Moreover, the trigger area had disappeared. Oxcarbazepine dose was stopped. Gabapentin was decreased to $600 \mathrm{mg}$ per day but could not be stopped.
One month after surgery, the patient reported that sensory disturbances had improved, concerning both tactile and thermal sensitivity. Two months after surgery, sensory testing monofilament and quantitative thermal assessment confirmed this subjective impression. Previously impaired thermal detection thresholds were normalized (Fig. 2, lower panel). Eighteen months after surgery the effect on pain and sensitivity remained the same.

\section{Discussion}

This case shows that sensory loss, resulting from peripheral nerve injury, has been reversed by MCS, early after surgery. Improvement of sensory loss in the painful zone after MCS has been previously reported in the literature [4, 5]. In one series, post-operative improvement of facial sensory discrimination was mentioned but not quantified in three patients, suffering from post-therapeutic neuralgia, geniculate neuralgia and post-stroke central pain [4]. Drouot et al. [5] reported improvement of thermal sensory thresholds by switching "on" MCS, in 8 of 18 patients having altered thermal sensory thresholds in the painful area. Changes in vibratory sensory thresholds were not significant. However, in this series, thermal changes were evaluated between 6 and 18 months after surgery without comparison with pre-operative status. These thermal sensory changes induced by MCS may be similar to those induced by repetitive transcranial magnetic stimulation (rTMS) $[6,7]$. RTMS has been shown to be efficient to treat neuropathic chronic pain and probably shares with MCS several of its mechanisms of action [8, 9]. However, the thermal sensory changes induced by rTMS were the opposite of those induced by MCS [8].

Fig. 1 Topography of pain and sensory loss, drawn by the patient. The arrow indicates the direction of intense paroxysmal attacks of pain, originating from the scar (dash), and triggered by touching the right side of the bridge of the nose (crosses). Hatched area shows the area of (tactile and thermal) sensory loss and lancinating pain

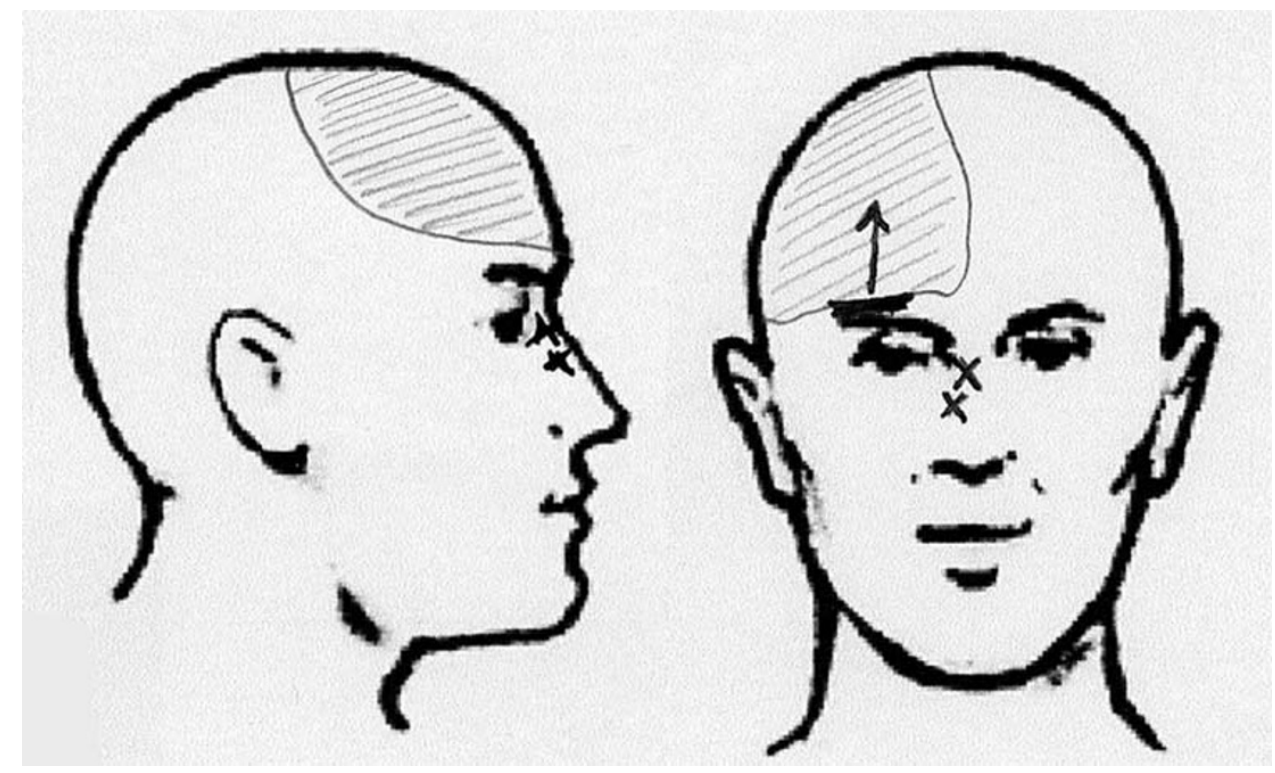




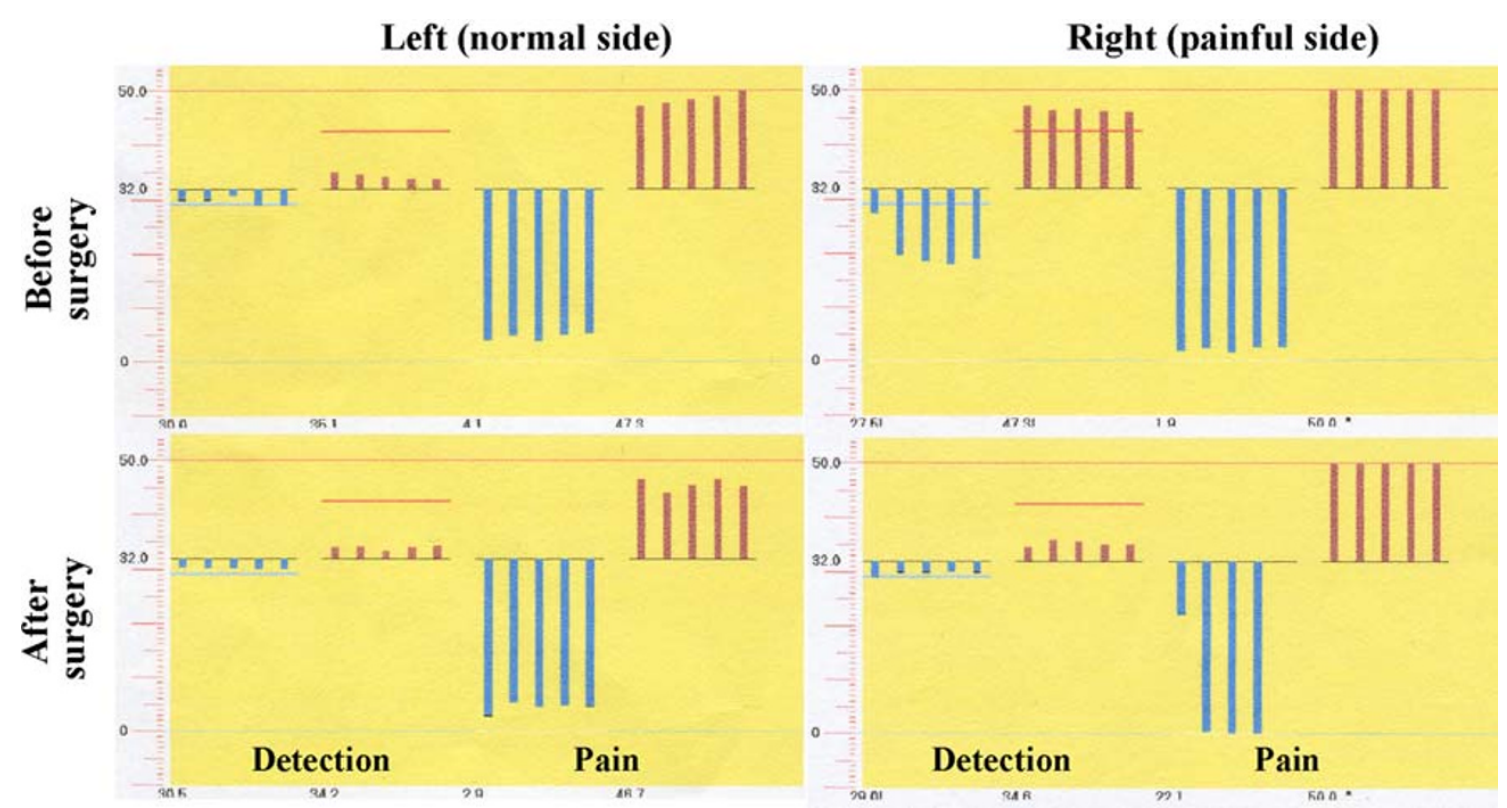

Fig. 2 Quantitative thermal detection and pain thresholds [cold in blue (columns 1, 3, 5 and 7) and heat in red (columns 2, 4, 6 and 8)], in the symptomatic (right) and uninjured (left) side, one month before (upper graphs) and 2 months after surgery (bottom graphs). The mean thresholds were calculated as the average value of five consecutive trials. Before surgery, both the cold and the heat detection thresholds were increased on the symptomatic side (20.4 and $46.6^{\circ} \mathrm{C}$, respectively) in comparison with the uninjured side $(29.8$

The present case is the first reported one in which thermal sensory changes are documented before and after surgery. Moreover, pain and sensory loss was clearly related to a peripheral nerve injury. The mechanisms leading to sensory restoration remain difficult to explain. Considering that nerve injury reduces the afferent input, the MCS improvement of sensory thresholds is quite surprising. It is very unlikely that MCS could enhance nerve growth to lead so quickly to sensory restoration, more than 2 years after initial injury. MCS could have acted by favoring plasticity and reorganization of the sensory cortex, but functional imaging studies have failed to demonstrate any activation of the somato-sensory cortex during or after MCS [10]. One hypothesis could be that the sensory impairment, initially due to the peripheral nerve injury, has been secondary maintained by central mechanisms, and that these central mechanisms have been reversed by MCS, leading to the sensory restoration. Deafferentation-induced neuronal hyperactivity [11] and reorganization of the sensory maps [12] in the thalamus are thought to disturb the control exerted by non-nociceptive sensory afferents on pain pathways and then contributing to the occurrence of neuropathic pain [13]. Drouot et al. [5] reported that pain was not reduced by MCS in patients in whom thermal sensory thresholds were not modified by switching "on" the MCS. MCS could both relieve pain and improve and $34.4^{\circ} \mathrm{C}$, respectively). After surgery, the thermal detection thresholds were normalized on the symptomatic side (29.8 and $35.3^{\circ} \mathrm{C}$, respectively) and similar to the uninjured side (30.2 and $34.1^{\circ} \mathrm{C}$ ). Normal values of cold and heat detection thresholds usually range between $29.3-32^{\circ} \mathrm{C}$ and $32-42.6^{\circ} \mathrm{C}$, respectively. Pre- and postoperative pain thermal thresholds were normal, confirming the absence of thermal allodynia (color in online)

sensory loss through changes in the altered thalamic sensory maps and/or by decreasing the thalamic neuronal hyperactivity $[1,14]$ interfering with sensory processing.

\section{Conclusion}

This case showed that MCS could restore tactile and thermal sensory loss resulting from peripheral nerve injury. Although the mechanisms leading to this effect remain unclear, this observation enhanced the hypothesis that MCS acts through modulation of the sensory processing. This observation has to be confirmed by systematic preand post-operative sensory testing with objective measurement of nerve function (e.g., blink reflex).

Conflict of interest None.

\section{References}

1. Tsubokawa T, Katayama Y, Yamamoto T et al (1991) Chronic motor cortex stimulation for the treatment of central pain. Acta Neurochir Suppl (Wien) 52:137-139

2. Fontaine D, Hamani C, Lozano A (2008) Efficacy and safety of motor cortex stimulation for chronic neuropathic pain: critical review of the literature. J Neurosurg. 7 November [Epub ahead of print] 
3. Rasche D, Ruppolt M, Stippich C et al (2006) Motor cortex stimulation for long-term relief of chronic neuropathic pain: a 10 year experience. Pain 121:43-52

4. Brown J, Pilitsis J (2005) Motor cortex stimulation for central and neuropathic facial pain: a prospective study of 10 patients and observations of enhanced sensory and motor function during stimulation. Neurosurgery 56:290-297

5. Drouot X, Nguyen J, Peschanski M et al (2002) The antalgic efficacy of chronic motor cortex stimulation is related to sensory changes in the painful zone. Brain 125:1660-1664

6. Summers J, Johnson S, Pridmore S et al (2004) Changes to cold detection and pain thresholds following low and high frequency transcranial magnetic stimulation of the motor cortex. Neurosci Lett 368:197-200

7. Johnson S, Summers J, Pridmore S (2006) Changes to somatosensory detection and pain thresholds following high frequency repetitive TMS of the motor cortex in individuals suffering from chronic pain. Pain 123:187-192

8. Wasserman E, Lisanby S (2001) Therapeutic applications of repetitive transcranial magnetic stimulation: a review. Clin Neurophysiol 112:1367-1377
9. Migita K, Uozumi T, Arita K et al (1995) Transcranial magnetic coil stimulation of motor cortex in patients with central pain. Neurosurgery 36:1037-1040

10. Garcia-Larrea L, Peyron R (2007) Motor cortex stimulation for neuropathic pain: from phenomenology to mechanisms. NeuroImage 37:S71-S79

11. Rinaldi P, Young R, Albe-Fessard D et al (1991) Spontaneous neuronal hyperactivity in the medial and intralaminar thalamic nuclei of patients with deafferentation pain. J Neurosurg 74:415421

12. Hua S, Garonzik I, Lee J et al (2000) Microelectrode studies of normal organization and plasticity of human somatosensory thalamus. J Clin Neurophysiol 17:559-574

13. Dostrovsky J (2000) Role of thalamus in pain. Prog Brain Res 129:245-257

14. Namba S, Nishimoto A (1988) Stimulation of internal capsule, thalamic sensory nucleus and cerebral cortex inhibited deafferentation hyperactivity provoked after gasserian ganglionectomy in cat. Acta Neurochirurgica (Suppl) 42:243-247 